$\mathrm{P}$ atients with severe heart failure in New York Heart Association (NYHA) functional class IIIIV, low left ventricular ejection fraction, and broad QRS have been shown to benefit from biventricular pacing in randomised crossover and parallel trials ${ }^{1-5}$ concerning symptomatic improvement such as exercise tolerance and quality of life (tables 1 and 2). The results are less clear for those with mild heart failure where further studies are needed. However, the evidence at large is based on sample size calculations made for six month follow up. ${ }^{1-5}$ Therefore it remains unknown whether the effects on biventricular pacing are long lasting in terms of years and whether morbidity and mortality is influenced. Although observational and controlled studies have demonstrated a reduced need for heart failure related hospitalisations during biventricular pacing, ${ }^{1-6}$ no study as yet with mortality as the primary outcome has been published. Therefore, whether cardiac resynchronisation therapy influences survival remains to be demonstrated in the recently concluded COMPANION and the ongoing CARE-HF study. These both have hospitalisations and mortality as the primary outcome..$^{7-8}$

The MUSTIC trial was not designed as a mortality study. ${ }^{1-3}$ Nevertheless, the two year survival rate in 104 patients on biventricular pacing was reported to be $80 \% .{ }^{9}$ The annual mortality rate was $10 \%$, which is lower than could be expected in these former NYHA class III patients. The causes of death were sudden $(40 \%)$ and heart failure death $(40 \%)$, and other causes $(20 \%)$. There is thus no direct evidence to date that the risk for sudden cardiac death is reduced by biventricular pacing. Recently the use of prophylactic implantable cardioverter-defibrillators (ICD) has been suggested in patients with coronary artery disease and reduced left ventricular ejection fraction. ${ }^{10}{ }^{11}$ In one study the benefit of a prophylactic defibrillator was greater in those with broad QRS. ${ }^{11}$ Therefore, it could be speculated that a prophylactic defibrillator should be added to a biventricular pacemaker in the treatment of the severe heart failure patient with intraventricular conduction disturbance. The aim of this paper is to discuss the evidence for this.

\title{
RISK OF ARRHYTHMIA
}

There is little to suggest that biventricular pacing can affect arrhythmia incidence. It could, however, be speculated that since biventricular pacing reduces the risk for bradycardia, the risk for bradyarrhythmia related sudden death-which accounts for a substantial proportion of sudden cardiac death in severe heart failure-would be reduced by pacing. ${ }^{12}{ }^{13} \beta$ Blocker treatment reduces sudden cardiac death by $40 \%$, suggesting that $\beta$ blockers reduce the risk for ventricular tachycardia. ${ }^{14}{ }^{15}$ Therefore, the introduction of a biventricular pacemaker could reduce the risk for sudden cardiac death both by preventing bradycardia and by enabling the introduction or up-titration of $\beta$ blocker treatment to optimal dose.

From the MUSTIC and the PATH-CHF studies, two randomised controlled trials, we know that heart rate is reduced and heart rate variability improved by long term biventricular pacing. ${ }^{3}{ }^{4} 16$ However, whether biventricular pacing per se prevents ventricular tachyarrhythmia remains doubtful. Only one small study indicates that the number of ventricular extra-beats, salvos, and duration of ventricular tachycardia were reduced during biventricular compared control pacing. ${ }^{17}$ However, interesting data have emerged from studies in which the primary indication for device implantation was an indication for defibrillator treatment. CONTAK-CD was a randomised crossover study of 490 patients with a classical indication for defibrillator treatment in combination with NYHA functional class II-IV heart failure and low ejection fraction. The first part of this study was a crossover comparison between three months each of ICD and biventricular pacing to ICD treatment alone concerning symptomatic improvement and safety. Of 581 patients included in the study, 490 were implanted: 245 were randomised to the combined treatment and the other 245 to ICD treatment alone. In contrast to biventricular pacing studies, somewhat more patients (66\%) had ischaemic heart disease as the underlying aetiology, and patients with NYHA class II were also included. ${ }^{18}$ The utilisation of antitachycardia pacing occurred in $9.4 \%$ of the patients in both groups. Shock treatment was administered in $19.2 \%$ of 
Table 1 Clinical effects of biventricular pacing

Improves:

Quality of life

Exercise tolerance

NYHA class

LV ejection fraction

LV dimensions

Heart rate variability

Reduces:

Total and heart failure related hospitalisations

Heart rate

LV, left ventricular; NYHA, New York Hear Association.

the 245 patients randomised to ICD and biventricular pacing treatment compared to $18 \%$ of those 245 subjects assigned to ICD treatment alone. Thus, biventricular pacing neither promoted nor reduced the delivery of ICD treatment in these patients.

\section{PROPHYLACTIC ICD TREATMENT \\ Cardiomyopathy}

The value of prophylactic ICD treatment in ischaemic and dilated cardiomyopathy has recently been studied. ${ }^{10}{ }^{11} 19$ The MADIT II trial included patients with prior myocardial infarction and low ejection fraction. No arrhythmia screening, either non-invasive or invasive, was required. Importantly, less than a third of patients had NYHA class III heart failure and patients in class IV were excluded from the study. Of the 1232 patients, 742 were randomly assigned to receive a prophylactic ICD and 490 were assigned to the control group.

Overall a $31 \%$ reduction in the risk of death at any interval during the four year follow up among patients in the defibrillator group compared to the conventional treatment group was found (fig 1). However, the benefit in terms of number of saved deaths by ICD was relatively small: 106 deaths $(14.2 \%)$ in the ICD arm compared to 97 deaths $(19.8 \%)$ in the conventional arm, corresponding to an absolute reduction of mortality of 5.6\%. Moreover, the survival benefit by ICD treatment became apparent only after nine months. Interestingly, hazard ratios in this study indicated that the greater the QRS width, the greater the survival benefit in the ICD arm.

This was further examined by Zareba who looked for noninvasive ECG criteria to identify patients who derive the most benefit from ICD treatment (oral communication, NASPE 2002). There were only two independent risk factors for prognosis: atrial fibrillation and QRS width. The three year mortality in 530 patients with QRS width of just 120 ms was $37 \%$ in the control group compared to $24 \%$ in the ICD

\begin{tabular}{l} 
Table 2 Indications for biventricular \\
pacing \\
\hline $\begin{array}{l}\text { Severe NYHA class III-IV heart failure despite } \\
\text { optimal drug treatment } \\
\text { Aetiology non-ischaemic or ischaemic heart } \\
\text { disease }\end{array}$ \\
QRS $>130 \mathrm{~ms}$ \\
LVEDD $>55 \mathrm{~mm}$ \\
LVEF $<35 \%$ \\
\hline LVEDD, left ventricular end diastolic diameter; \\
LVEF, left ventricular ejection fraction.
\end{tabular}

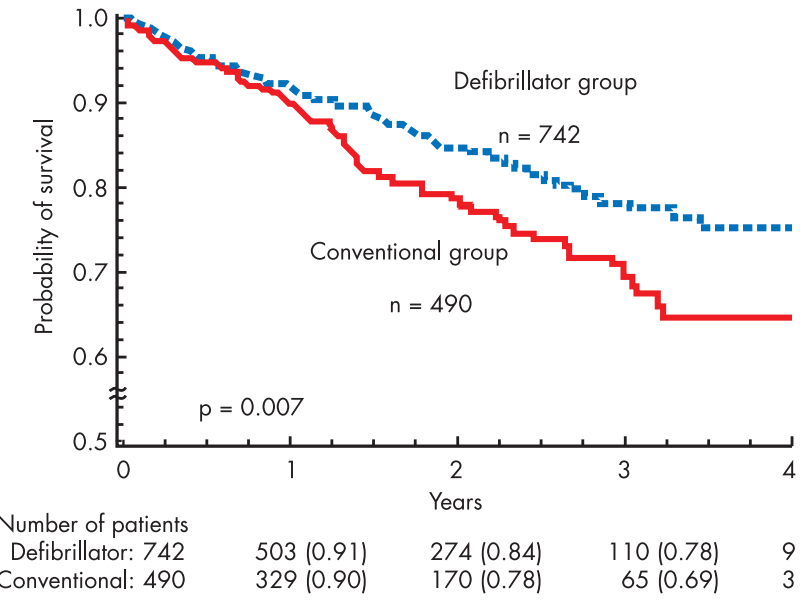

$$
H R=0.69(P=0.016) \rightarrow 31 \% \text { reduction in mortality }
$$

Figure 1 Survival in 104 patients with previous myocardial infarction assigned to prophylactic ICD treatment $(n=742)$ or to the control group ( $\mathrm{n}=490$ ) in the MADIT-II study. Reproduced from Moss et al," with permission of the Massachusetts Medical Society.

group - a 49\% reduction in mortality resulting from ICD treatment. In those 364 patients with a QRS above $120 \mathrm{~ms}$ the three year mortality was 53\% in the control group compared to $21 \%$ in the ICD group, translating to a mortality reduction of $63 \%$. Thus, the reduction in mortality by ICD treatment was substantially greater in those with a wide QRS than in the MADIT-II population as a whole. Moreover, except for QRS width, there was no indication that noninvasive arrhythmia screening such as heart rate variability was of any value in mortality risk prediction. Therefore, it seems clear that a prophylactic ICD in combination with biventricular pacing is indicated in patients fulfilling the entry criteria for both the MADIT II and for biventricular pacing.

In contrast, the beneficial value of prophylactic ICD treatment for patients with dilated cardiomyopathy remains unknown. Only one study so far aimed at studying exclusively such patients. ${ }^{19}$ The CAT trial was a primary prevention study in 104 dilated cardiomyopathy patients followed for a mean of 5.5 years. ${ }^{19}$ The study compared the survival benefit of ICD treatment to control treatment in patients with recent onset dilated cardiomyopathy, ejection fraction below 30\%, and NYHA class II-III heart failure. Patients with NYHA class IV or symptomatic brady- or tachycardia were excluded. The primary end point in the study was one year total mortality. The study was terminated

\section{Abbreviations}

CARE-HF: Cardiac Resynchronisation in Heart Failure CAT: Cardiomyopathy Trial COMPANION: Comparison of Medical Therapy, Pacing and Defibrillation in Chronic Heart Failure ICD: implantable cardioverter-defibrillator

MADIT: Multi-center Autonomic Defibrillator Implantation Trial

MUSTIC: Multisite Stimulation in Cardiomyopathy NYHA: New York Heart Association PATH-CHF: Pacing Therapies of Congestive Heart Failure 


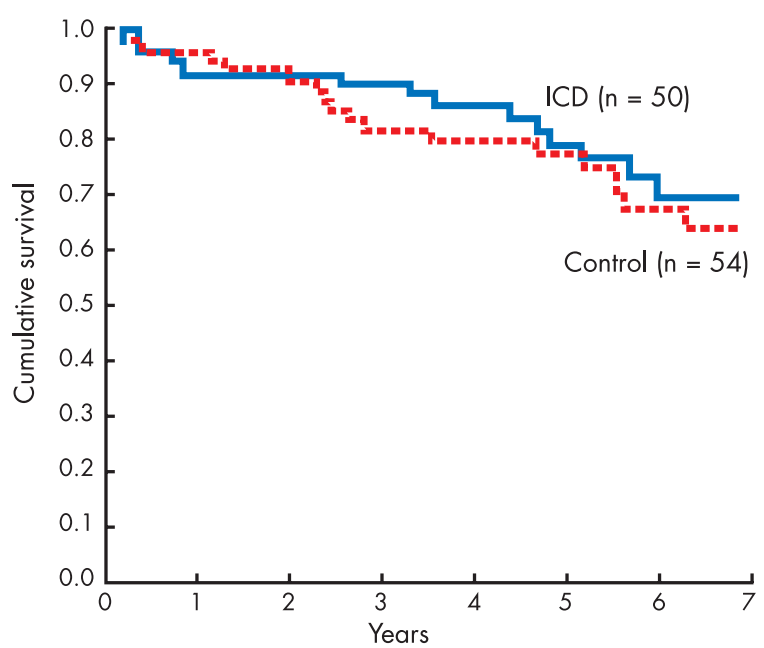

Number remaining at risk

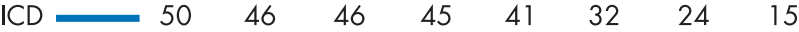

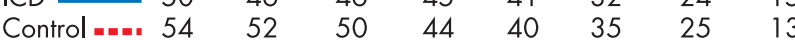

Figure 2 Survival rates in 104 patients with dilated cardiomyopathy assigned to prophylactic ICD treatment or to the control group in the CAT study. Reproduced from Bänsch et $a{ }_{1}{ }_{1}^{19}$ with permission.

early because the all cause mortality at one year did not reach the expected $30 \%$ but was only $5.6 \%$. The mean follow up time was 22 months. Drug treatment at baseline comprised angiotensin converting enzyme inhibitors in $96 \%$ of the subjects and $\beta$ blockers in only $4 \%$. No report of amiodarone treatment is given. Survival rates of the 50 patients assigned to ICD treatment and those 54 patients assigned to the control group did not differ significantly between groups (fig 2). In those assigned to ICD treatment the survival rate in those who experienced ventricular tachycardia during follow up was in fact worse than in those who did not. This indicates that activity in electrical disease paralleled overall infirmity. Moreover, the presence of non-sustained ventricular tachycardia on Holter ECG did not impact survival in the control group. In conclusion, the findings from this study do not indicate a survival benefit with prophylactic ICD treatment in patients with dilated cardiomyopathy and reduced left ventricular function $(<35 \%)$.

\section{Severe heart failure}

It is not self evident that the evidence from these prophylactic ICD trials for primary prevention of sudden death can be applied automatically to severe heart failure patients eligible for biventricular pacing. In MADIT-II only $30 \%$ of patients had NYHA class III-IV heart failure and 50\% had a QRS duration > $>120 \mathrm{~ms}$, with no information as to which proportion of these patients had both abnormalities. Thus, maximally $30-50 \%$ of them would have had a classical indication for biventricular pacing. Since at least 50\% of patients in published studies on biventricular pacing had dilated cardiomyopathy as the underlying disorder, the evidence for the usefulness of a prophylactic ICD in biventricular pacing candidates is clearly called for. ${ }^{1-5}$ Moreover, Duncan and colleagues recently reported that patients with underlying dilated cardiomyopathy benefit more from biventricular pacing regarding symptom reduction and reverse left ventricular remodelling than those with underlying ischaemic heart disease. ${ }^{20}$ In the CAT study, 35\% of patients would have been candidates for biventricular pacing based on the functional class criteria, and $36 \%$ had left bundle branch block. Therefore, the question of whether a biventricular pacemaker should always be combined with a defibrillator should be answered by studies in which the main indication for device implantation was cardiac resynchronisation treatment.

So far, only the COMPANION trial has included an ICD arm. $^{7}$ In the COMPANION study 2200 severe heart failure patients with a QRS duration above $120 \mathrm{~ms}$ were enrolled and randomised to receive optimal medical treatment alone, optimal medical treatment and biventricular pacing, or optimal medical treatment and biventricular pacing combined with an ICD in a 1:2:2 fashion. The primary end point was combined-that is, total mortality and all cause hospitalisations. The study was conducted on the assumption that an estimated event rate in the control arm of $40 \%$ should be reduced by $25 \%$ in both device arms. This required 2200 patients to be enrolled and to be followed for two years. The study was terminated early when 1600 patients had been enrolled because the primary end point had been reached in both device arms. The primary end point was significantly reduced by $20 \%$ both in the biventricular pacing arm and the biventricular pacing plus ICD arm. The secondary end point of total mortality alone was significantly reduced by $42 \%$ in the biventricular pacing plus ICD arm only. These results will have to be confirmed, but if true they point in favour of combined therapy for biventricular pacing candidates. Moreover, the results from the CARE-HF study, which are expected to emerge in 2004, will answer the remaining question of whether biventricular pacing alone reduces mortality. ${ }^{8}$

\section{CONCLUSION}

Although heart rate and sympathetic nerve activity is reduced by cardiac resynchronisation therapy, there is no evidence that biventricular pacing prevents or provokes ventricular arrhythmias. Prophylactic ICD treatment is indicated in prior myocardial infarction patients with broad QRS. No solid evidence for the usefulness of a prophylactic ICD in dilated cardiomyopathy patients exist to date. Whether all patients with a classical indication for biventricular pacing should also receive a prophylactic ICD thus remains to be determined in ongoing trials. ${ }^{7}$

\section{ACKNOWLEDGEMENTS}

Supported by the Swedish Heart and Lung Association.

\section{REFERENCES}

1 Cazeau S, Leclercq C, Lavergne T, for the Multisite Stimulation in Cardiomyopathy, et al. Effects of multisite biventricular pacing in patients with heart failure and intraventricular conduction delay. N Engl J Med $2001 ; 12: 873-80$

- Important randomised crossover study on the clinical effects of biventricular pacing in sinus rhythm patients.

2 Leclercq C, Walker S, Linde C, et al. Comparative effects of permanent biventricular and right univentricular pacing in heart failure patients with chronic atrial fibrillation. Eur Heart J 2002;23:1780-7.

- Only randomised crossover study on the clinical effects of biventricular pacing in atrial fibrillation patients.

3 Linde C, Leclercq C, Rex S, et al. Long-term benefits of biventricular pacing in congestive heart failure. Results from the MUSTIC (multisite stimulation in cardiomyopathy) study. J Am Coll Cardiol 2002;40:111-18.

- One year follow up of clinical effects of biventricular pacing in sinus rhythm and atrial fibrillation patients.

4 Auricchio A, Stellbrink C, Sack S, et al. Chronic effects of hemodynamically optimized cardiac resynchronisation therapy on patients with heart failure and ventricular conduction delay. J Am Coll Cardiol 2002;39:2026-33.

- One year follow up of clinical effects of biventricular pacing in sinus rhythm patients. 
5 Abraham WT, Fisher WG Smith $\mathrm{AL}$, et al for the Miracle Investigators and Coordinators. Multicentre InSync Randomised Clinical Evaluation (MIRACLE). Results of a randomised, double blind, controlled trial to assess cardiac resynchronisation therapy in heart failure patients. J Am Coll Cardiol 2001;38:595-6.

- Parallel six month comparison of biventricular pacing and optimal medical treatment on symptomatic improvement in $\mathbf{4 5 3}$ patients.

6 Braunschweig F, Linde C, Gadler F, et al. Reduction of hospital days by biventricular pacing. Eur J Heart Failure 2000;2:399-406.

7 Bristow MR for the Companion Steering Committee and Clinical Investigators. Heart failure management using implantable devices for ventricular resynchronisation: comparison of medical therapy, pacing and defibrillation in chronic heart failure (COMPANION) trial. J Cardiac Failure 2000;6:276-85.

8 Cleland JG, Daubert JC, Erdmann E, et al. The Care-HF study (cardiac resynchronisation in heart failure study): rationale, design and end-points. Eur J Heart Fail 2001;3:481-9

9 Daubert C, Linde C, Cazeau S, et al. Mortality in patients included in the MUSTIC study: long term (>2 years) follow up. Pacing Clin Electrophysiol 2002;24:558A.

10 Buxton AE, Lee KL, Fisher JD, et al for the Multicentre Unsustained Tachycardia Trial Investigators. A randomised study of the prevention of sudden death in patients with coronary artery disease. N Engl J Med 1999:341:1882-90.

- Randomised study on the value of prophylactic ICD treatment in patients with ischaemic heart disease and left ventricular dysfunction.

11 Moss A, Zareba W, Hall WJ, et al. Prophylactic implantation of a defibrillator in patients with myocardial infarction and reduced ejection fraction. N Engl J Med 2002;346:877-83.

- Randomised study on the value of prophylactic ICD treatment in patients with previous myocardial infarction and left ventricular dysfunction.
12 Luu M, Stevenson WG, Stevenson LW et al. Diverse mechanisms of unexpected cardiac arrest in advanced heart failure. Circulation 1989;80:1675-80.

13 Bayes de Luna A, Coumel $P$, et al. Ambulatory sudden cardiac death: mechanisms of production of fatal arrhythmia on the basis of data from 157 cases. Am Heart J 1989;1 17:151-9.

14 The CIBIS II Investigators and Committees. The cardiac insufficiency bisoprolol study II (CIBIS II): a randomised trial. Lancet 1999;353:9-13.

- This study on patients with NYHA class III-IV heart failure assesses the efficacy of bisoprolol compared to placebo on all cause mortality.

15 MERIT-HF Study Group. Effects of metoprolol CR/XL in chronic heart failure: metoprolol $\mathrm{CR} / \mathrm{XL}$ randomised intervention trial in congestive heart failure (MERIT.HF). Lancet 1999;353:2001-7.

- Randomised study of patients with NYHA class II-IV heart failure assessing the efficacy of metoprolol compared to placebo on all cause mortality.

16 Alonso C, Ritter $\mathrm{P}$, Leclercq $\mathrm{C}$, et al. Biventricular pacing improves heart rate variability in patients with chronic systolic heart failure and intraventricular conduction delay. Am J Cardiol 2003;91:1144-6.

17 Walker S, Levy TM, Rex S, et al. Usefulness of suppression of ventricular arrhythmia by biventricular pacing in severe congestive heart failure. Am J Cardiol 2000;86:231-3.

18 Kumar V, Higgins SL, Putz EJ, et al. Effect of chronic resynchronisation therapy on ICD therapies. Pacing Clin Electrophysiol 2002;24:558.

19 Bänsch D, Antz M, Boczor S, et al for the CAT Investigators. Primary prevention of sudden cardiac death in idiopathic dilated cardiomyopathy. Circulation 2002; 105:1453-8

20 Duncan $A$, Wait $D$, Gibson D, et al. Left ventricular remodelling and haemodynamic effects of multisite biventricular pacing in patients with left ventricular dysfunction and activation disturbances in sinus rhythm: sub-study of the MUSTIC (multisite stimulation in cardiomyopathy) trial. Eur Heart J 2003;24:430-41.

- Important study on the key effects of biventricular pacing on left ventricular remodelling. 